\title{
Pulsed operation of a miniature scalar optically- pumped magnetometer
}

\author{
Vladislav Gerginov, ${ }^{1,2 *}$ Sean KRZYZeWSKI, ${ }^{2}$ SVenja KNAPPE ${ }^{2,3}$ \\ ${ }^{1}$ Time and Frequency Division, NIST, 325 Broadway, Boulder, CO 80305, USA \\ 2University of Colorado, Boulder, $\mathrm{CO} 80309$ \\ ${ }^{3}$ QuSpin Inc., 2011 Cherry Street, Louisville CO 80027 \\ *Corresponding author: Vladislav.Gerginov@nist.gov
}

\begin{abstract}
A scalar magnetic field sensor based on a millimeter-size ${ }^{87} \mathbf{R b}$ vapor cell is described. The magnetometer uses nearly co-propagating pump and probe laser beams, amplitude modulation of the pump beam, and detection through monitoring the polarization rotation of the detuned probe beam. The circularly-polarized pump laser resonantly drives a spin precession in the alkali atoms at the Larmor frequency. A modulation signal on the probe laser polarization is detected with a lock-in amplifier. Since the Larmor precession is driven all-optically, potential crosstalk between sensors is minimized. And since the pump light is turned off during most of the precession cycle large offsets of the resonance, typically present in a single-beam Bell-Bloom scheme, are avoided. At the same time, relatively high sensitivities can be reached even in millimeter-size vapor cells: The magnetometer achieves a sensitivity of $1 \mathrm{pT} / \mathrm{Hz}^{1 / 2}$ in a sensitive volume of $16 \mathrm{~mm}^{3}$, limited by environmental noise. When a gradiometer configuration is used to cancel the environmental noise, the magnetometer sensitivity reaches $300 \mathrm{fT}^{-\mathrm{Hz}^{1 / 2}}$. We systematically study the dependence of the magnetometer performance on the optical duty cycles of the pump light and find that better performance is achieved with shorter duty cycles, with the highest values measured at $1.25 \%$ duty cycle. (c) 2015 Optical Society of America
\end{abstract}

OCIS codes: (020.0020) Atomic and molecular physics; (130.6010) Sensors;(300.6210) Spectroscopy, atomic.

http://dx.doi.org/10.1364/AO.99.099999

\section{INTRODUCTION}

Optically-pumped magnetometers (OPMs) have generated renewed interest over the last 15 years, in part due to their increased sensitivity, when operated in the spin-exchange relaxation free (SERF) regime [1]. Good fundamental sensitivity was experimentally demonstrated [2], rivaling that of the best superconducting quantum interference device (SQUID) magnetometers [3]. SERF magnetometers have been used for a variety of applications ranging from imaging the brain [4-9] and fetal heart $[10,11]$ to nuclear magnetic resonance $[12,13]$ and magnetic resonance imaging $[14,15]$. These applications require very highperformance sensors that can get very close to the samples and can tolerate measurements inside a shielded environment.

For many applications outside shielded environments, especially those where the sensors are not stationary, scalar OPMs are often favored because they are less sensitive to mechanical noise of the mounting platform, which leads to changes of their orientation with respect to the ambient field. First implemented in the 1970s [16], they have since developed into rugged commercial products. They are routinely used for magnetic anomaly detection (MAD), unexploded ordinance detection (UXO), and geophysical surveying for archeology [17] or mineral and oil exploration [18]. Recently, airborne mapping with unmanned autonomous vehicles (UAV) planes has become possible with smaller, lighter and lower power magnetometers and has generated renewed interest in device miniaturization.
During the last ten years, substantial progress has been made on reducing the size of OPMs, since smaller sensors can allow for lower power consumption. Discharge lamps have been replaced by lowpower diode lasers [19]. Furthermore, less power is needed to heat smaller vapor cells [20]. Vacuum packaged atomic devices have shown to consume powers as little as $10 \mathrm{~mW}$ [21].

The background environmental noise level in an unshielded environment could be above $10 \mathrm{pT} / \mathrm{Hz}^{1 / 2}$ below $10 \mathrm{~Hz}$, especially in urban environments [22]. While this would limit the performance of magnetometers, much of this noise can be suppressed using multiple sensors in a gradiometer configuration [23, 24]. Therefore, when operated in noisy environments, smaller magnetometer size and better sensitivity can allow for gradiometers with shorter baselines.

Simultaneously, there has been substantial effort in improving the sensitivity of miniaturized scalar OPMs. For DC magnetic field detection with continuous pumping, the fundamental limit set by spin-exchange collisions, photon shot noise, and quantum back-action noise is on the order of $0.5 \mathrm{fT} \mathrm{cm}^{3 / 2} / \mathrm{Hz}^{1 / 2}$ [25]. In pulsed operation and using quantum non-demolition measurement, a scalar gradiometer sensitivity of $0.54 \mathrm{fT} / \mathrm{Hz}^{1 / 2}$ has been experimentally demonstrated with multi-pass cells in a sensitive volume of $350 \mathrm{~mm}^{3}$ [26]. Under continuous operation, several groups have shown sensitivities below $1 \mathrm{pT} / \mathrm{Hz}^{1 / 2}$ in centimeter size cells [24,27-30]. Microfabricated scalar sensors have reached sensitivities of $5 \mathrm{pT} / \mathrm{Hz}^{1 / 2}$ in a volume of $2 \mathrm{~mm}^{3}$ with a bandwidth of $1 \mathrm{kHz}$ [31]. Using the effect of light narrowing, sensitivities 
of $42 \mathrm{fT} / \mathrm{Hz}^{1 / 2}$ in an active volume of $9.3 \mathrm{~mm}^{3}$ and $10 \mathrm{fT} / \mathrm{Hz}^{1 / 2}$ in $50 \mathrm{~mm}^{3}$ were predicted, if the noise were photon shot noise-limited $[28,29]$. In an actual measurement, a good sensitivity of $320 \mathrm{fT} / \mathrm{Hz}^{1 / 2}$ was reached in a relatively small cell of $50 \mathrm{~mm}^{3}$ [32]. It was operated as an all-optical Bell-Bloom magnetometer [33], when the laser intensity noise was cancelled with a secondary light monitor.

Scalar magnetometers have in most cases used field coils or modulated pump light to drive the Larmor precession. All-optical schemes are often favored when cross-talk between sensors can be an issue, since they do not produce external magnetic fields except to the one caused by the atomic magnetization precession. When implemented as a single-beam version, they can also have reduced heading errors compared to their coil driven $\mathrm{Mx}$ counterpart, for example, that arise from misalignment of the RF coil with the light axis in combination with the nonlinear Zeeman effect. Nevertheless, many scalar OPM sensors in applications use the Mx scheme, partially because it can be very tedious to optimize the control electronics for Bell-Bloom operation due to large offsets of the signal due to the pump modulation. Here we investigate an all-optical Bell-Bloom scheme with separate, nearly-parallel pump and probe laser beams in which the offsets are largely suppressed using polarimeter detection and intensity modulation at a subharmonic of the Larmor frequency. We investigate the magnetometer performance as a function of duty cycle. In contrast to single-beam experiments [32], the two-beam setup favors a smaller duty cycle which reduces power broadening of the resonance. Finally, we demonstrate a scalar magnetometer with a $16 \mathrm{~mm}^{3}$ active volume in a pulsed mode of operation. A sensitivity as low as $300 \mathrm{fT} / \mathrm{Hz}^{1 / 2}$ is achieved, and a potential photon shot noise-limited sensitivity below $200 \mathrm{fT} / \mathrm{Hz}^{1 / 2}$ is demonstrated. A gradiometer configuration was used to assess the magnetometer sensitivity.

\section{EXPERIMENTAL SETUP}

The scalar magnetometer uses a cylindrical glass vapor cell with inner diameter and length of $3.3 \mathrm{~mm}$ and $3.8 \mathrm{~mm}$, respectively. The cell is filled with isotopically pure ${ }^{87} \mathrm{Rb}$ and contains nitrogen as a buffer gas with a pressure of $66.7 \mathrm{kPa}$ (500 Torr). The cell is mounted in a cylindrical aluminum housing ( $25.4 \mathrm{~mm}$ diameter, with $5 \mathrm{~mm}$ diameter opening for the cell) with on-axis optical ports. The aluminum is heated with flexible heaters placed several centimeters away. The resonant optical depth of the cell is 0.7 at $358 \mathrm{~K}\left(85^{\circ} \mathrm{C}\right)$. The cell is placed inside a magnetic shield chamber to reduce the effects of ambient magnetic fields on the sensor evaluation. A static magnetic field $\mathrm{B}_{0}=14 \mu \mathrm{T}$ is applied at the cell position, perpendicular to the optical axis, created by a pair of Helmholtz coils driven by a DC current source with a current noise below $50 \mathrm{pA} / \mathrm{Hz}^{1 / 2}$ (corresponding to $30 \mathrm{fT} / \mathrm{Hz}^{1 / 2}$ at $24 \mathrm{~mA}$ ) at frequencies above $10 \mathrm{~Hz}$ except for $60 \mathrm{~Hz}$ harmonics.

The atomic spin-precession is resonantly driven all optically [33]. In the past, frequency, amplitude, or polarization modulation has been used [34]. The scalar magnetometer described here is based on the BellBloom optically-driven spin-precession scheme [33], where a spin precession in a static magnetic field is induced by a pump laser beam, amplitude-modulated (AM) at the Larmor frequency or a subharmonic of it. We use amplitude instead of frequency modulation to minimize the noise resulting from the presence of pump light during the precession cycle. The spin precession causes rotation of the linear polarization of a far-detuned probe beam $[35,36]$. The polarization rotation is detected with a balanced polarimeter. The magnetometer implementation is similar to the sensor geometry which use nonlinear magneto-optical rotation (NMOR) and amplitude modulation $[37,38]$. In this work, the polarization rotation is due to a precessing atomic orientation, in contrast to Ref. $[37,38]$ where atomic alignment was used. For this reason, it is difficult to make performance comparisons with work based on atomic alignment.
In this experiment, we use a modulation at half of the Larmor frequency, and phase detection at the Larmor frequency. The modulation at half of the Larmor precession frequency reduces the power broadening from the pump laser, at the expense of a slightly reduced signal size $(\sim 10 \%)$. Direct detection of scattered light from the pump laser does not create a background signal, and the phase detection is performed at a higher frequency, where the technical noise is reduced.

The magnetometer uses two diode lasers (pump and probe) with wavelengths near the ${ }^{87} \mathrm{Rb} \mathrm{D} 1$ resonance $(795 \mathrm{~nm})$. Both lasers have linewidths on the order of $1 \mathrm{MHz}$. The magnetometer setup is shown in Fig. 1.

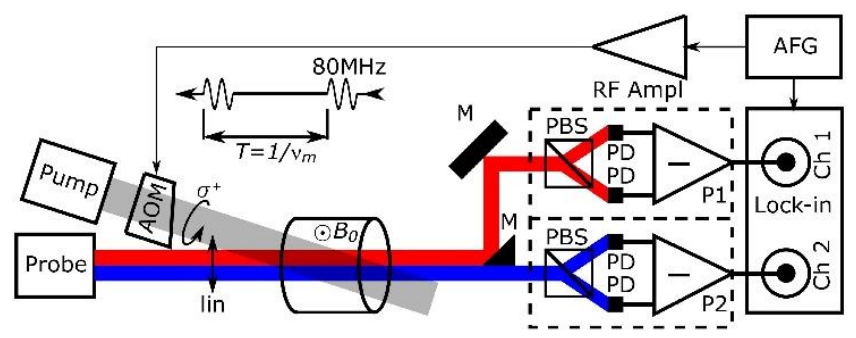

Fig. 1. Sensor setup. The amplitude-modulated pump beam propagates through the vapor cell at a small angle with respect to the probe beam in a plane perpendicular to the static magnetic field $\mathrm{B}_{0}$ inside the vapor cell. The probe beam is split geometrically for two-path detection by the polarimeters P1 and P2 and demodulated with a lock-in amplifier. PBS - Glan-Taylor polarizers, PD - photodetector, M - mirror.

The pump laser light passes through an acousto-optic modulator $(\mathrm{AOM})$, and the first diffraction order is coupled into a polarizationmaintaining (PM) fiber (not shown). The collimated fiber output has a waist of $3 \mathrm{~mm}$ and is circularly polarized. The pump beam frequency is in resonance with the buffer gas pressure-broadened $\sim 11.5 \mathrm{GHz}$ wide ${ }^{87} \mathrm{Rb} \mathrm{D} 1$ resonance, which is red-detuned by $\sim 1.2 \mathrm{GHz}$ from the unperturbed $F=2-F^{\prime}=1$ optical transition and corresponds to a wavelength of $780.249 \mathrm{~nm}$. An $80 \mathrm{MHz} \mathrm{RF}$ carrier signal for the AOM is generated with an arbitrary function generator (AFG), and is amplitudemodulated at $100 \%$ and $49 \mathrm{kHz}$ with a pulse train waveform of adjustable duty-cycle. The $80 \mathrm{MHz}$ pulses delivered to the AOM and the AOM response allows optical pulse lengths of $1 \mu$ s or longer, with an average power of more than $500 \mu \mathrm{W}$. The average pulse train power is varied by adjusting the amplitude of the $80 \mathrm{MHz}$ carrier signal. The AM frequency $v_{m}$ is also used for phase detection with a digital lock-in amplifier.

The probe laser beam is coupled into a PM fiber. The collimated output has a $3 \mathrm{~mm}$ waist diameter and is linearly polarized. The probe laser is red-detuned from the unperturbed $F=2-F^{\prime}=1$ optical transition resonance by $\sim 9.2 \mathrm{GHz}$ and corresponds to a wavelength of $780.266 \mathrm{~nm}$.

The pump and the probe laser beams propagate almost parallel in a plane perpendicular to $\mathrm{B}_{0}$. The beams cross at a small angle $(\sim 5 \mathrm{mrad})$ at the cell position to allow for independent spatial detection of the probe beam only. The probe beam is geometrically split, and the two halves of the probe beam are detected by independent polarimeters (P1 and P2 in Fig. 1) using Glan-Taylor polarizers and balanced photodetectors. The polarimeter signals are sent to the two inputs of the lock-in amplifier for phase-sensitive detection at half the Larmor frequency. A gradiometer configuration can be realized by subtracting the two polarimeter signals electronically using the two inputs of the lock-in amplifier. It is implemented here to suppress the environmental noise, stemming mostly from nearby components. 


\section{RESULTS}

\section{A. Resonance linewidth and magnetometer bandwidth}

The amplitude-modulated circularly-polarized pump beam creates an atomic polarization through the process of optical pumping, which in turn leads to a rotation of the linear probe polarization. For the geometry of Fig. 1, the presence of the static magnetic field $\mathrm{B}_{0}$, orthogonal to the direction of the circularly-polarized pump beam, causes the atomic polarization to precess at the Larmor frequency around the direction of $\mathrm{B}_{0}$ in a plane perpendicular to $\mathrm{B}_{0}$. The precession leads to a modulation of the probe polarization, which is detected by the polarimeters. A resonant condition occurs when the Larmor frequency matches the AM frequency $\nu_{m}$ or any of its harmonics. In this work, the Larmor frequency was twice as high as the modulation frequency, and the phase detection was performed at the second harmonic of $v_{\mathrm{m}}$.

The resonance is shown in Fig. 2 (curves (a) and (c)) as in-phase and quadrature lock-in output of polarimeter P1 as a function of the magnetic static field $\mathrm{B}_{0}$ in the interval $14 \pm 2.6 \mu \mathrm{T}$. No offset subtraction was necessary for the signals.

To calibrate the magnetic field $\mathrm{B}_{0}$ scale, the modulation frequency $\nu_{\mathrm{m}}$ was changed by $1 \mathrm{kHz}$ from $49 \mathrm{kHz}$ to $50 \mathrm{kHz}$. Therefore, the resonance occurred at a higher magnetic field, corresponding to a Larmor frequency change from $98 \mathrm{kHz}$ to $100 \mathrm{kHz}$, as can be seen from the horizontal offset of curve (b) in Fig. 2. The Larmor frequency shift of $2 \mathrm{kHz}$ corresponds to a magnetic field change of $0.28 \mu \mathrm{T}$ (using the gyromagnetic ratio $\gamma=2 \pi \times 7 \mathrm{~Hz} / \mathrm{nT}$ for ${ }^{87} \mathrm{Rb}$ ). The calculated magnetic field change was used to calibrate the lower horizontal axis.

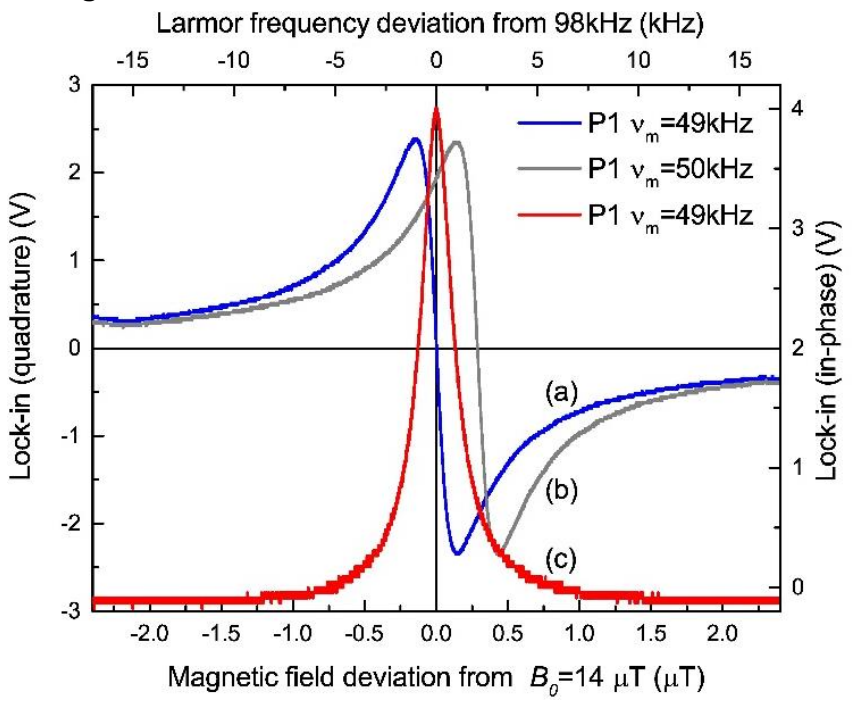

Fig. 2. Lock-in amplifier output as a function of the magnetic field $\mathrm{B}_{0}$ for polarimeter P1. The modulation frequency $\nu_{m}$ was $49 \mathrm{kHz}$ (curves (a), quadrature signal and (c), in-phase signal) and $50 \mathrm{kHz}$ (curve (b), quadrature signal). The Larmor frequency is given (top $\mathrm{x}$-scale) as a deviation from $98 \mathrm{kHz}$.

The full width at half maximum of the spin precession resonance is $0.28 \mu \mathrm{T}$ (or $2 \mathrm{kHz}$ ), determined by a combination of optical power broadening, spin exchange broadening $\left(\sim 500 \mathrm{~Hz}\right.$ at $358 \mathrm{~K}$ or $\left.85^{\circ} \mathrm{C}\right)$, optical power broadening ( $\sim 1 \mathrm{kHz}$ from the probe and $\sim 500 \mathrm{~Hz}$ from the pump beam), buffer gas pressure $(\sim 20 \mathrm{~Hz}$ at $66.7 \mathrm{kPa})$, and wall collisions $(\sim 30 \mathrm{~Hz})[39]$.

The magnetometer bandwidth is determined by the transverse relaxation time $T_{2}$ of the atoms, and is therefore directly related to the width of the spin-precession resonance. Measurement of the magnetometer frequency response to a fixed-amplitude sine-wave magnetic field modulation at frequencies in the range of $40 \mathrm{~Hz}$ to $2.5 \mathrm{kHz}$ was used to calculate the magnetometer bandwidth. The resulting $3 \mathrm{~dB}$ magnetometer bandwidth was $750 \mathrm{~Hz}$, in agreement with the experimentally measured spin-precession resonance linewidth of $2 \mathrm{kHz}$.

\section{B. Magnetometer performance}

The magnetometer performance was optimized against the alkali atoms density (determined by the cell temperature), as well as frequency detuning and power of the pump and probe beams. These parameters were kept constant and optimized in a range very similar to those of the single-beam Bell-Bloom magnetometer.

The magnetometer performance was studied as a function of duty cycle (ratio of the pumping duration to the duration of the modulation period) of the pump laser, where the average pump laser power per Larmor period was kept the same for each duty cycle. In a single-beam Bell-Bloom magnetometer a duty cycle of 0.5 usually gives the best performance [32]. With separated pump and probe beams, it is found here that shorter pump pulses give better results.

Figure 3 shows the dependence of the measured magnetometer resonance amplitude (middle) and resonance linewidth (bottom) when the pump duty cycle was varied between $1.25 \%$ and $50 \%$. It can clearly be seen that the amplitude increases and linewidth decreases with short duty cycles. The probe beam power of $4.35 \mathrm{~mW}$, and the pump power of $0.73 \mathrm{~mW}$ were kept the same for all duty cycle measurements. Since the average power is kept the same, and the power broadening contributes to only $25 \%$ of the resonance linewidth, it is expected that the resonance linewidth would depend weakly on the duty cycle. On the other hand, the increase of the duty cycle leads to a wider spread of precession angles in the plane perpendicular to $\mathrm{B}_{0}$, caused by the simultaneous actions of the optical pumping and Larmor precession, and results in decreased signal amplitude. For $50 \%$ duty cycle, the precession angle is $2 \pi$, and the signal amplitude is zero. As seen from Fig. 3, the resonance linewidth broadens almost linearly with the duty cycle for small duty cycles, while the resonance amplitude remains nearly constant and drops off rapidly after a duty cycle of $20 \%$.

The broadening and amplitude reduction of the spin precession resonance with longer pump pulse duration also leads to a significant loss of sensitivity (top of Figure 3). The magnetometer sensitivity was determined as follows: A FFT spectrum analyzer measures the voltage noise (in units of $\mathrm{V}_{\mathrm{rms}} / \mathrm{Hz}^{1 / 2}$ ) at the output of the lock-in amplifier. The voltage noise is converted into magnetic field noise through the slope of the dispersion curve (Fig. 2 (a)) near the center of the resonance. The magnetic field noise as a function of frequency is normalized by dividing the magnetic field noise frequency spectrum by the measured magnetometer frequency response. The sensitivity is estimated from the normalized magnetic field noise level. It can be seen from Figure 3 that the sensitivity degrades rapidly at duty cycles longer than $20 \%$. 


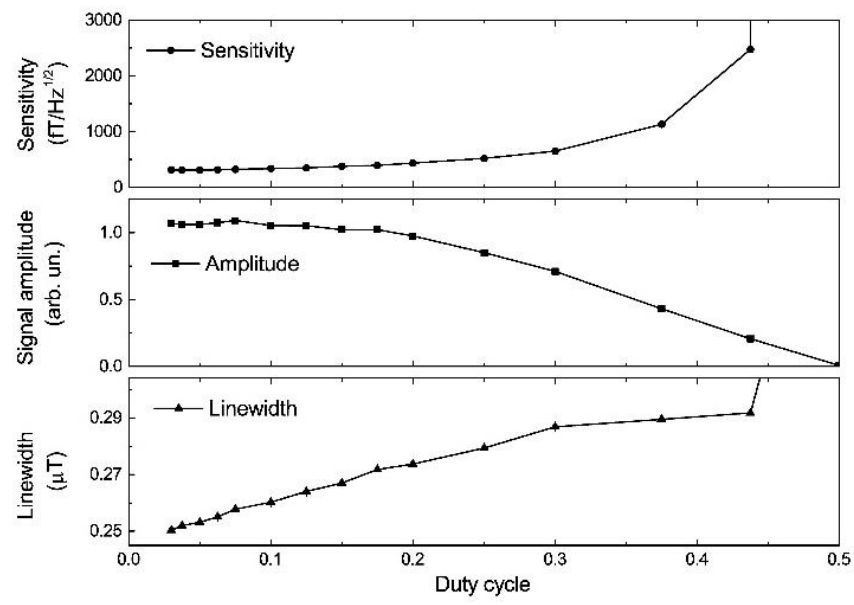

Fig. 3. Single-channel sensor sensitivity (top, gradiometer mode), resonance amplitude (middle) and width (bottom), as a function of the AM duty cycle. The average pump laser power per Larmor period was kept the same for each duty cycle.

The magnetic field noise measured by a single-channel magnetometer (using the signal from polarimeter P1 only) is shown in Fig. 4, curve (a). A similar result is obtained using the signal from polarimeter P2. From the noise level, a sensitivity of $1 \mathrm{pT} / \mathrm{Hz}^{1 / 2}$ for frequencies between 100 and $500 \mathrm{~Hz}$ (Fig. 4, curve (a)) is estimated. The sensitivity degrades at higher frequencies due to the limited magnetometer bandwidth. The peaks are due to magnetic field noise in the laboratory. The origin of sensitivity degradation below $100 \mathrm{~Hz}$ is discussed below.

A magnetometer sensitivity is limited by a combination of environmental (Earth's field noise, bias field noise, Johnson noise from nearby conducting surfaces) and intrinsic noise (photon-shot noise, spin-projection noise). For that reason, it is important to shield the magnetometer from noisy magnetic field environment during its sensitivity evaluation.

When the environmental magnetic field noise limits the sensitivity, the intrinsic single-channel magnetometer sensitivity can be assessed using the gradiometer mode of operation. In this mode, common-mode noise (such as environmental magnetic field noise) is largely rejected, and the measured noise level is determined by the uncorrelated intrinsic noise of the single sensors. However, field inhomogeneities over the vapor cell volume are not removed, and could lead to a decreased sensitivity.

By applying a sinusoidal modulation to the static magnetic field $\mathrm{B}_{0}$, a common-mode rejection ratio (CMRR) of 20 was measured in the gradiometer mode in the range $40 \mathrm{~Hz}$ to $2 \mathrm{kHz}$. Assuming the intrinsic noise of the single sensors is uncorrelated, the single-channel magnetometer sensitivity was estimated by dividing the gradiometer signal by square root of two. In the gradiometer mode, sensitivities around $300 \mathrm{fT} / \mathrm{Hz}^{1 / 2}$ for frequencies between 40 and $500 \mathrm{~Hz}$ are demonstrated (Fig. 4, curve (b)).

The single polarimeter noise floor (electronic and probe laser photon shot noise, Fig. 4, curve (c)) is determined by blocking the pump laser beam and measuring the noise spectrum at the output of the lock-in amplifier. The noise spectrum at magnetic fields far detuned from resonance is also at the same level.

The calculated photon shot noise level for a single polarimeter is shown with the orange dash-dotted curve, and is below $200 \mathrm{fT} / \mathrm{Hz}^{1 / 2}$ at frequencies between 40 and $100 \mathrm{~Hz}$. It is slightly above the measured noise floor with the pump laser off, which could be due to imperfect photocurrent calibration. Since the measured noise floor (curve (c)) includes the photon shot-noise of the probe laser, it shows that it should be possible to reach sensitivities below $200 \mathrm{fT} / \mathrm{Hz}^{1 / 2}$ with a singlechannel magnetometer.

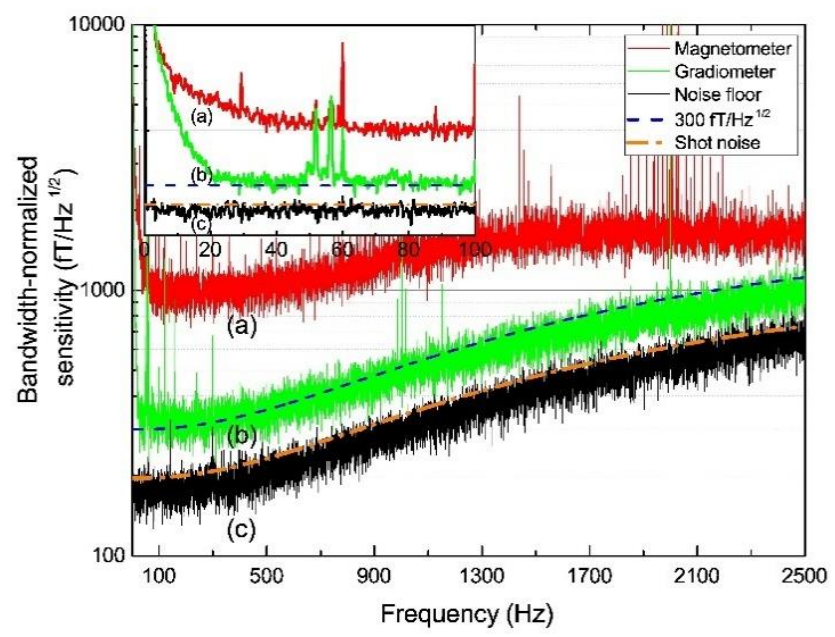

Fig. 4. Sensor sensitivity as a function of measurement frequency. Curve (a) - single channel, magnetometer mode; curve (b) - single channel, gradiometer mode; curve (c) - single channel, system noise (pump laser off). Calculated sensitivities of $300 \mathrm{fT} / \mathrm{Hz}^{1 / 2}$ and the photon shot-noise sensitivity, are shown with dashed and dashed-dotted lines, respectively. The inset shows the data in the frequency range $0-100 \mathrm{~Hz}$.

The dashed curve in Fig. 3 represents a sensitivity of $300 \mathrm{fT} / \mathrm{Hz}^{1 / 2}$ normalized for the magnetometer bandwidth. It matches the intrinsic single-channel magnetometer performance in gradiometer mode for frequencies above $1 \mathrm{kHz}$. At frequencies below $500 \mathrm{~Hz}$, the measured noise spectrum lies slightly above the measured gradiometer sensitivity. The additional low-frequency noise could be caused by residual localized environmental magnetic field fluctuations not completely canceled by the gradiometer, or a deviation from 1-st order filter response.

We suspect that local inhomogeneities in the magnetic field noise caused by Johnson noise of the aluminum housing. The contribution from the housing was estimated at the cell position using the formalism in Ref. [40], resulting in the same order of magnitude as the noise measured with the magnetometer below $500 \mathrm{~Hz}$, and has a cutoff frequency on the order of $100 \mathrm{~Hz}$. The aluminum housing is in contact with the vapor cell which has sub-millimeter wall thickness and it is expected that the local field noise is not entirely correlated between the two polarimeter channels, each using a geometric half of the laser beam propagating through the vapor cell. We therefore expect slightly better performance if the cell heater was replaced with a non-conductive version.

One advantage of the single-beam Bell-Bloom magnetometer is the low cross-talk due to the all-optical scheme. Another advantage is the possibility of reducing heading errors from misalignment of the RF coil, which is of importance for applications of moving platforms [41]. The currentsetup uses a small angle between pump and probe and with that introduces some potential heading errors. This could be mediated by overlapping the pump and probe completely. It could be done, for example, by shifting the wavelength of the probe light to the ${ }^{87} \mathrm{Rb} \mathrm{D} 2$ resonance at $780 \mathrm{~nm}$. The setup could then be simplified and its footprint reduced by using the same PM fiber for the probe and the pump beams. The necessary polarizations could be created with a multi-order wave plate, and the pump beam blocked by an interference 
filter [6, 24]. Alternatively, a single laser could be used as pump and probe, where the frequency and polarization of the laser is modulated simultaneously in a way that is serves as the pump during the first short part of the cycle and as the probe for the rest. Data is only collected during the "probe cycle".

\section{CONCLUSIONS}

A pulsed scalar Bell-Bloom magnetometer with $\mathrm{Rb}$ vapor cell with $16 \mathrm{~mm}^{3}$ internal volume is described. Resonant pump light is circularly polarized and pulsed at half the Larmor frequency. A continuous probe beam detects the atomic precession on a balanced polarimeter. It is found that shorter duty cycles of the pump light lead to better performance, most likely due to reduced spread of polarization angles due to the simultaneous action of optical pumping and precession. The magnetometer has a bandwidth of $750 \mathrm{~Hz}$. A magnetometer sensitivity below $1 \mathrm{pT} / \mathrm{Hz}^{1 / 2}$ was demonstrated. The measured sensitivity was limited by environmental magnetic field noise, most likely due to Johnson noise of the cell heating housing. The magnetic field noise was reduced by using two sensors in a gradiometer mode, achieving intrinsic single-channel sensitivity below $300 \mathrm{fT} / \mathrm{Hz}^{1 / 2}$ in a sensitive volume of $16 \mathrm{~mm}^{3}$. A photon shot noise-limited sensitivity below $200 \mathrm{fT} / \mathrm{Hz}^{1 / 2}$ seems possible.

Funding Information. National Institute of Health (NIH) (1R01EB019440, R01NS094604).

Acknowledgment. The authors thank Dr. Roger Brown and Dr. Abigail Perry for their valuable comments on the manuscript. This work is a contribution of NIST, an agency of the US government, and is not subject to copyright.

\section{References}

1. J. C. Allred, R. N. Lyman, T. W. Kornack, and M. V. Romalis, "High Sensitivity Atomic Magnetometer Unaffected by Spin-Exchange Relaxation," Phys. Rev. Lett. 89, 130801 (2002).

2. H. B. Dang, A. C. Maloof, and M. V. Romalis, "Ultrahigh sensitivity magnetic field and magnetization measurements with an atomic magnetometer," Applied Physics Letters 97, 151110 (2010).

3. J.-H. Storm, D. Drung, M. Burghoff, and R. Körber, "A modular, extendible and field-tolerant multichannel vector magnetometer based on current sensor SQUIDs," Superconductor Science and Technology 29, 094001 (2016).

4. H. Xia, A. Ben-Amar Baranga, D. Hoffman, and M. V. Romalis, "Magnetoencephalography with an atomic magnetometer," Applied Physics Letters 89, 211104 (2006).

5. T. H. Sander, J. Preusser, R. Mhaskar, J. Kitching, L. Trahms, and S. Knappe, "Magnetoencephalography with a chip-scale atomic magnetometer," Biomedical Optics Express 3, 981 (2012).

6. C. Johnson, P. D. D. Schwindt, and M. Weisend, "Magnetoencephalography with a two-color pump-probe, fibercoupled atomic magnetometer," Applied Physics Letters 97, 243703 (2010).

7. K. Kim, S. Begus, H. Xia, S.-K. Lee, V. Jazbinsek, Z. Trontelj, and M. V. Romalis, "Multi-channel atomic magnetometer for magnetoencephalography: A configuration study," Neurolmage 89, 143 (2014).

8. V. K. Shah and R. T. Wakai, "A compact, high performance atomic magnetometer for biomedical applications," Physics in Medicine and Biology 58, 8153 (2013).

9. K. Kamada, D. Sato, Y. Ito, H. Natsukawa, K. Okano, N. Mizutani, and T. Kobayashi, "Human magnetoencephalogram measurements using newly developed compact module of high-sensitivity atomic magnetometer," Japanese Journal of Applied Physics 54, 026601 (2015).

10. R. Wyllie, M. Kauer, G. S. Smetana, R. T. Wakai, and T. G. Walker, "Human magnetoencephalogram measurements using newly developed compact module of high-sensitivity atomic magnetometer," Physics in Medicine and Biology 57, 2619 (2012).

11. O. Alem, T. H. Sander, R. Mhaskar, J. LeBlanc, H. Eswaran, U. Steinhoff, Y. Okada, J. Kitching, L. Trahms, and S. Knappe, "Fetal magnetocardiography measurements with an array of microfabricated optically pumped magnetometers," Physics in Medicine and Biology 60, 4797 (2015).

12. I. M. Savukov and M. V. Romalis, "NMR Detection with an Atomic Magnetometer," Phys. Rev. Lett. 94, 123001 (2005).

13. T. Theis, P. Ganssle, G. Kervern, S. Knappe, J. Kitching, M. P. Ledbetter, D. Budker, and A. Pines, "Parahydrogen-enhanced zero-field nuclear magnetic resonance," Nat. Phys. 7, 571 (2011).

14. I. Savukov, V. Zotev, P. Volegov, M. Espy, A. Matlashov, J. Gomez, and R. K. Jr., "MRI with an atomic magnetometer suitable for practical imaging applications," Journal of Magnetic Resonance 199, 188 (2009).

15. T. Oida, M. Tsuchida, and T. Kobayashi, "Direct Detection of Magnetic Resonance Signals in Ultra-Low Field MRI Using Optically Pumped Atomic Magnetometer with Ferrite Shields: Magnetic Field Analysis and Simulation Studies," IEEE Transactions on Magnetics 48, 2877 (2012).

16. M. Livanov, A. Kozlov, A. Korinevski i, V. Markin, and S. Sinel'nikova, "Recording of human magnetic fields," Doklady Akademii nauk SSSR 238, 253-256 (1978).

17. A. David, M. Cole, T. Horsley, N. Linford, P. Linford and L. Martin, "A rival to Stonehenge? Geophysical survey at Stanton Drew, England," Antiquity 78, 341 (2004).

18. M. N. Nabighian, V. J. S. Grauch, R. O. Hansen, T. R. LaFehr, Y. Li, J. W. Peirce, J. D. Phillips and M. E. Ruder, "The historical development of the magnetic method in exploration," Geophysics 70, 6 (2005)

19. S. Groeger, A. S. Pazgalev, and A. Weis, "Comparison of discharge lamp and laser pumped cesium magnetometers," Applied Physics B 80, 645 (2005).

20. V. Shah, S. Knappe, P. D. D. Schwindt, and J. Kitching, "Subpicotesla atomic magnetometry with a microfabricated vapour cell," Nat Photon 1,649 (2007).

21. M. J. Mescher, R. Lutwak, and M. Varghese, "An ultra-low-power physics package for a chip-scale atomic clock," in "The 13th International Conference on Solid-State Sensors, Actuators and Microsystems", vol. 1, pp. 311-316 (2005).

22. "SQUID Sensors: Fundamentals, Fabrication and Applications", ed. H. Weinstock, Springer Science+Business Media (1995)

23. D. A. Keder, D. W. Prescott, A. W. Conovaloff, and K. L. Sauer, "An unshielded radio-frequency atomic magnetometer with subfemtoTesla sensitivity," AIP Advances, 4, 127159 (2014).

24. G. Bevilacqua, V. Biancalana, P. Chessa, and Y. Dancheva, "Multichannel optical atomic magnetometer operating in unshielded environment," Applied Physics B 122, 1 (2016).

25. S. Smullin, I. Savukov, G. Vasilakis, R. Ghosh, and M. Romalis, "Lownoise high-density alkali-metal scalar magnetometer," Phys. Rev. A 80, 033420 (2009).

26. D. Sheng, S. Li, N. Dural, and M. V. Romalis, "Subfemtotesla Scalar Atomic Magnetometry Using Multipass Cells," Phys. Rev. Lett. 110, 160802 (2013).

27. G. Bison, N. Castagna, A. Hofer, P. Knowles, J.-L. Schenker, M. Kasprzak, H. Saudan, and A. Weis, "A room temperature 19channel magnetic field mapping device for cardiac signals," Applied Physics Letters 95, 173701 (2009).

28. T. Scholtes, V. Schultze, R. IJsselsteijn, S. Woetzel, and H. G. Meyer, "Light-narrowed optically pumped M-x magnetometer with a miniaturized Cs cell," Phys. Rev. A 84 (2011). 
29. V. Schultze, B. Schillig, R. IJsselsteijn, T. Scholtes, S. Woetzel and R. Stolz, "An Optically Pumped Magnetometer Working in the Light-Shift Dispersed Mz Mode", Sensors 17, 3, (2017).

30. W. Wasilewski, K. Jensen, H. Krauter, J. J. Renema, M. V. Balabas, and E. S. Polzik, "Quantum Noise Limited and Entanglement-Assisted Magnetometry," Phys. Rev. Lett. 104, 133601 (2010).

31. P. D. D. Schwindt, B. Lindseth, S. Knappe, V. Shah, J. Kitching, and L.A. Liew, "Chip-scale atomic magnetometer with improved sensitivity by use of the Mx technique," Applied Physics Letters 90, 081102 (2007).

32. V. Schultze, R. IJsselsteijn, T. Scholtes, S. Woetzel, and H.-G. Meyer, "Characteristics and performance of an intensity-modulated optically pumped magnetometer in comparison to the classical $\mathrm{Mx}$ magnetometer," Optics Express 20, 14201 (2012).

33. W. E. Bell and A. L. Bloom, "Optically driven spin precession," Phys. Rev. Lett. 6, 280 (1961).

34. Z. D. Grujic and A. Weis, "Atomic magnetic resonance induced by amplitude-, frequency-, or polarization-modulated light," Phys. Rev. A

35. 88, 012508 (2013).Y. Takahashi, K. Honda, N. Tanaka, K. Toyoda, K. Ishikawa, and

T. Yabuzaki, "Quantum nondemolition measurement of spin via the paramagnetic Faraday rotation," Phys. Rev. A 60, 4974 (1999).

36. V. Shah, G. Vasilakis, and M. V. Romalis, "High Bandwidth Atomic

37. Magnetometery with Continuous Quantum Nondemolition Measurements," Phys. Rev. Lett. 104, 013601 (2010).W. Gawlik, L. Krzemien, S. Pustelny, D. Sangla, J. Zachorowski, M. Graf, A. O. Sushkov, D. Budker, "Nonlinear magneto-optical rotation with amplitude modulated light", Appl. Phys. Lett. 88, 131108 (2006).

38. S. Pustelny, A. Wojciechowski, M. Gring, M. Kotyrba, J. Zachorowski, W. Gawlik, " Magnetometry based on nonlinear magneto-optical rotation with amplitude-modulated light", J. Appl. Phys. 103, 063108 (2008).

39. J. Vanier and C. Audoin, "The Quantum Physics of Atomic Frequency Standards", Institute of Physics Publishing (1989)

40. S.-K. Lee and M. V. Romalis, "Calculation of magnetic field noise from high-permeability magnetic shields and conducting objects with simple geometry," Journal of Applied Physics 103, 084904 (2008).

41. A. Bloom, "Principles of operation of the rubidium vapor magnetometer," Appl. Opt., vol. 1, pp. 61-68 (1962). 